Regards sur l'économie allemande

Bulletin économique du CIRAC

$67 \mid 2004$

Varia

\title{
Consommation : les gros budgets des petits Allemands
}

\section{Isabelle Bourgeois}

\section{(2) OpenEdition}

1 Journals

Édition électronique

URL : https://journals.openedition.org/rea/3812

DOI : 10.4000/rea.3812

ISBN : 978-2-8218-0830-0

ISSN : 1965-0787

\section{Éditeur}

CIRAC

Édition imprimée

Date de publication : 1 juillet 2004

Pagination : 34-36

ISSN : 1156-8992

Référence électronique

Isabelle Bourgeois, "Consommation : les gros budgets des petits Allemands ", Regards sur l'économie allemande [En ligne], 67 | juillet 2004, mis en ligne le 08 octobre 2009, consulté le 21 septembre 2021. URL : http://journals.openedition.org/rea/3812 ; DOI : https://doi.org/10.4000/rea.3812

Ce document a été généré automatiquement le 21 septembre 2021.

(c) CIRAC 


\title{
Consommation : les gros budgets des petits Allemands
}

\author{
Isabelle Bourgeois
}

\section{$73 €$ d'argent de poche par mois en moyenne}

1 Les jeunes Allemands ont le porte-monnaie bien garni. En deux ans, ils ont vu leur 'revenu disponible' progresser de... $24 \%$. En 2003, ils disposaient de 20,43 milliards $€$ au total, contre 16,44 milliards e seulement en 2001. A l'argent de poche s'ajoutent les 'gratifications' à Noël ou pour l'anniversaire et, pour une large partie des plus de 16 ans, les premiers salaires : petits jobs ou rémunération dans le cadre de l'apprentissage. Chacun des 11,28 millions de 6-19 ans recensés outre-Rhin a ainsi en poche $73 €$ par mois au minimum, selon les résultats de l'enquête marketing KidsVerbraucherAnalyse 2003, menée par divers groupes de presse jeunesse, dont Bauer et Springer. Ils sont nettement plus riches que les jeunes Français qui, selon la dernière enquête de l'INSEE, disposent de $163 €$ par an en moyenne à l'âge de 11 ans et de $427 €$ à 17 ans.

Les finances des 6-19 ans en Allemagne (2003)

\begin{tabular}{|l|l|l|l|l|l|}
\hline & 'Revenu' mensuel & + Noël & + Anniversaire & Epargne & Libre disposition $*$ ) \\
\hline En moyenne & $73 €$ & $95 €$ & $84 €$ & $762 €$ & $77 \%$ \\
\hline Filles & $64 €$ & $86 €$ & $78 €$ & $764 €$ & $76 \%$ \\
\hline $6-9$ ans & $11 €$ & $54 €$ & $45 €$ & $487 €$ & $48 \%$ \\
\hline $10-12$ ans & $21 €$ & $69 €$ & $63 €$ & $580 €$ & $73 \%$ \\
\hline $13-15$ ans & $38 €$ & $93 €$ & $81 €$ & $670 €$ & $86 \%$ \\
\hline
\end{tabular}




\begin{tabular}{|l|l|l|l|l|l|}
\hline $16-19$ ans & $164 €$ & $120 €$ & $118 €$ & $1222 €$ & $96 \%$ \\
\hline Garçons & $81 €$ & $103 €$ & $90 €$ & $761 €$ & $77 \%$ \\
\hline $6-9$ ans & $13 €$ & $51 €$ & $47 €$ & $543 €$ & $46 \%$ \\
\hline $10-12$ ans & $20 €$ & $66 €$ & $66 €$ & $510 €$ & $75 \%$ \\
\hline $13-15$ ans & $41 €$ & $110 €$ & $83 €$ & $623 €$ & $87 \%$ \\
\hline $16-19$ ans & $217 €$ & $170 €$ & $152 €$ & $1249 €$ & $97 \%$ \\
\hline
\end{tabular}

Source des données : KidsVerbraucherAnalyse 2003, Egmont Ehapa/Bauer/Axel Springer. Enquête réalisée en janvier et février 2003 auprès d'un panel représentatif de 2643 enfants et adolescents de 6 à 19 ans, et de leurs parents. *) « Mon fils/ma fille peut disposer librement de son argent de poche... toujours, presque toujours, souvent».

\section{Noël : poupées pour les filles, autos pour les garçons}

2 Les filles ont moins d'argent à leur disposition que les garçons. On trouve là le reflet des inégalités entre les sexes et, pour les plus de 16 ans, celui des disparités salariales liées au choix des métiers : les filles s'orientent plus volontiers vers les métiers notamment du tertiaire, considérés comme 'féminins', mais moins rémunérateurs aussi (voir REA 49/00). La disparité dans le montant des cadeaux de Noël ou d'anniversaire s'explique, elle, en grande partie par le type de cadeau désiré et son prix. Chez les 6-12 ans, on constate la traditionnelle opposition entre les poupées ou peluches (en tête des souhaits des filles avec $14 \%$, suivies des montres bracelet avec $13 \%$ ) d'un côté et, de l'autre, les voitures et circuits électriques ( $18 \%$ des garçons) ou les trains électriques $(16 \%)$.

\section{Garçons et filles rêvent de jeux électroniques}

Quant à la 'fracture numérique' entre les sexes, elle tend à se réduire chez les enfants même si elle reste nette: alors que les garçons sont $32 \%$ à posséder des jeux électroniques (et $30 \%$ à rêver de s'en faire offrir), les filles les rattrapent avec respectivement $23 \%$ et $17 \%$. Mais les enfants ne sont pas encore nombreux à posséder leur propre PC : $13 \%$ des garçons et $10 \%$ des filles seulement. $38 \%$ des premiers rêvent donc d'en avoir un, pour $25 \%$ des secondes. Alors, où vont-ils jouer? Chez eux, pour l'essentiel (50\% des foyers sont équipés), ou alors chez les copains (39\%) - une tendance en nette progression depuis 2001 (20\%).

\section{$89 \%$ des adolescents ont un portable}

Autre moyen de communication 'sexué' : le téléphone portable, que possèdent $19 \%$ des filles et $15 \%$ seulement des garçons. Si la fonction de contrôle parental est moins prononcée qu'en France, les petites Allemandes apprennent elles aussi très tôt à cultiver le lien social. Tous sexes confondus, l'intérêt pour le portable augmente bien 
sûr avec l'âge : seulement $7 \%$ des 6-9 ans en possèdent un, mais déjà $29 \%$ des 10-12 ans, puis $66 \%$ des 13-15 ans jusqu'à un taux d'équipement de $89 \%$ chez les 16-19 ans. La demande est loin d'être saturée : tous ne sont pas encore équipés, et l'effet mode est particulièrement prononcé dans cette catégorie de produits à innovation rapide. Pour ce qui est des factures, les parents (et les adolescents) se montrent prudents : $67 \%$ des 13-19 ans utilisent une carte pre-paid. La responsabilisation s'accroît avec l'âge : la carte recule chez les 16-19 ans (58\% seulement) au profit du contrat en nom propre (24\% des plus âgés).

\section{Prescripteurs, en Allemagne aussi}

5 Les jeunes Allemands accordent beaucoup d'importance aux marques. Et leurs parents cèdent volontiers à leurs désirs : à $54 \%$ pour les baskets, à $49 \%$ pour les vêtements, à $46 \%$ pour les jeans, et à $50 \%$ voire plus pour les cartables / sacs à dos ou les fournitures scolaires. Comme leurs homologues français, ils les 'gâtent' ainsi pour leur éviter de s'exclure socialement. Particulièrement 'branchés' mode, les petits Allemands sont également d'importants prescripteurs pour leurs parents, qu'ils conseillent dans l'achat de vêtements (72\%), de chaussures (70 \%) ou d'équipements sportifs.

\section{Vacances familiales en Allemagne}

6 Très informés (près des trois quarts des adolescents ont leur propre téléviseur, et autant surfent régulièrement sur Internet), ils ont aussi voix prépondérante dans le choix des lieux et types de vacances. Dès l'âge de 13 ans, ils sont plus de $50 \%$ à codécider "toujours ou souvent" de la destination. Cela se comprend: ils n'ont généralement le droit de partir seuls en vacances qu'après 16 ans (62\% des filles mais seulement $58 \%$ des garçons, jugés moins responsables en l'occurrence). En tête des destinations familiales : l'Allemagne (28\%), suivie de l'Italie (13\%) et de la Turquie (8\%). A noter que $15 \%$ des familles ne sont pas parties en vacances en 2003.

Comment vivent les Allemands de 6-19 ans?

\begin{tabular}{|l|l|l|}
\hline & $6-12$ ans & $13-19$ ans \\
\hline Total & 5,38 millions & 5,90 millions \\
\hline Filles & 2,62 millions & 2,86 millions \\
\hline Garçons & 2,76 millions & 3,04 millions \\
\hline Scolarité & & \\
\hline Ecole primaire & $58 \%$ & - \\
\hline Hauptschule & $15 \%$ & $11 \%$ \\
\hline Realschule & $12 \%$ & $28 \%$ \\
\hline
\end{tabular}




\begin{tabular}{|l|l|l|}
\hline Gymnasium & $6 \%$ & $29 \%$ \\
\hline Gesamtschule & - & $6 \%$ \\
\hline Autre & $3 \%$ & $10 \%$ \\
\hline Non encore scolarisés & $6 \%$ & - \\
\hline En formation professionnelle & - & $13 \%$ \\
\hline Dans la vie active & - & $3 \%$ \\
\hline Frère ou sœur & & \\
\hline non & $33 \%$ & n.c. \\
\hline oui & $67 \%$ & $64 \% *)$ \\
\hline Comment vivent-ils? & $85 \%$ & $94 \%$ \\
\hline Parents propriétaires (maison ou appartement) & $43 \%$ & $47 \%$ \\
\hline Ont leur propre chambre & & \\
\hline
\end{tabular}

Source des données : KidsVerbraucherAnalyse 2003, Ehapa/Bauer/Axel Springer. Enquête réalisée en janvier et février 2003 auprès d'un panel représentatif de 2643 enfants et adolescents de 6 à 19 ans, et de leurs parents. *) Au moins un frère ou une sœur.

\section{Les filles lisent Bravo, les garçons, Computer Bild}

7 Que font-ils des $1811 €$ dont ils disposent en moyenne par an? Ils aiment consommer. Les 6-12 ans s'achètent en priorité des bonbons et des glaces, mais aussi des périodiques (Junior et Micky Maus en tête) ; et ils dépensent le reste pour des sodas ou des sorties au fast food. Plus de la moitié des 13-19 ans placent en tête de leurs achats la presse jeunesse. Leur titre préféré est alors Bravo, mais avec une distinction selon le sexe : si les adolescentes plébiscitent ce titre (27,4\% contre $19,2 \%$ pour les garçons), les adolescents lisent plus que les filles le magazine Computer Bild (21,7 \% contre seulement $5,7 \%)$. Filles et garçons se distinguent également par la structure de leurs autres dépenses : cosmétiques, vêtements et chaussures pour les premières, jeux vidéo et électronique pour les seconds. Mais (comme en France) plus rien ne les distingue en matière de $\mathrm{CD}$, de restauration rapide, de cinéma et de téléphone.

\section{Les adolescents épargnent pour s'acheter une voiture}

8 Si tous aiment dépenser, ils sont tout de même 82 \% à mettre de l'argent de côté. Le livret d'épargne domine ( $83 \%$ des jeunes). En 2003, ils ont ainsi épargné 8,60 milliards $€$ au total. Les plus petits se constituent un budget 'jouets' : les garçons plutôt pour un portable, un vélo ou des jeux électroniques; les filles plutôt pour la mode. Les plus grands épargnent en prévision prioritairement du permis de conduire $(26 \%$ des 
garçons et $31 \%$ des filles de plus de 13 ans) et de l'achat d'une voiture (respectivement $21 \%$ et $25 \%$ ). S'ils partagent ainsi le rêve d'autonomie des jeunes Français, leurs priorités reflètent aussi la très grande mobilité privée et professionnelle des Allemands. (IB)

INDEX

Mots-clés : consommation, niveau de vie, pouvoir d'achat, jeune, groupe social, argent, revenu 